\title{
Analog SKED
}

\author{
F. E. BUTLER \\ University of Iowa, Iowa City, Iowa 52242
}

\begin{abstract}
Analog data can now be handled by SKED. Through the use of F3s, input, output, decisions, and analyses can be performed on analog data. A new command has been added to SKED. The trade off between number of boxes and resolution is discussed.
\end{abstract}

The state notational system (Snapper, Knapp, \& Kushner, 1970) has been used to describe experiments (Snapper, 1973; Lyon, Note 3, Michael, Note 4). The notational system has also laid the groundwork for computer languages: SKED [Snapper, Knapp, Kushner, \& Kadden (Note 5)] ; ACT (Millenson, 1971); and SCAT (Polson, 1973). The notation system makes use of discrete events.

SKED has previously been used to gather electrophysiological data. To date, the data have been discrete: peak of the $\mathrm{R}$ wave in cardiac recordings (Kadden, Note 2), zero crossings of brain waves in alpha conditioning (Snapper, 1973), and single unit conditioning (Butler, Note 1). Until now, analog signals have not been dealt with by SKED. The lack of an analog package has caused some to generate special-purpose programs instead of using SKED (Fowler, 1974; Millenson, Kehoe, Tait, \& Gormezano, 1973; Tait \& Gormezano, 1974).

After analog data are converted to digital form, they are discrete and, as such, easily fit into the state notational system. All that is needed is a routine to control the analog to digital converter. This may be added through the use of the F3 function (Snapper \& Hamilton, 1974; Snapper \& Walker, 1971). Decisions and analyses, based on the analog data, can be made by the use of additional F3s. Output can be in analog form after conversion from digital form. This is done by yet another $\mathrm{F} 3$ that controls the digital to analog converter.

\section{ANALOG I/0}

\section{F3(A/D)}

The analog to digital conversion routine is the heart of the analog package. The routine assumes a multiplexer and 8-bit conversion, left justified. The form of the F3 is:

F3(A/D starting address, channel number, counter low, counter high, counter low, $Z$ end)

The arguments in the F3 are as follows: The channel

This research was conducted while the author was supported by a NSP traineeship Grant MH 10641 . number is specified. The $\mathrm{A} / \mathrm{D}$ value is deposited into counter low. An example of this may be seen in State Set 2 of Figure 1. Every 2 msec the routine is called, Channel 1 is sample, and the $A / D$ value is deposited in Counter 1. After $2 \mathrm{msec}$, the $A / D$ value goes into Counter 2 , and so on. When the $A / D$ value goes into Counter 150, a Z3 is issued which causes a transition to State 1. The next time the routine is called, the $A / D$ value goes into Counter 1 .

\section{F3(D/A)}

The digital to analog $(D / A)$ routine can display on an oscilloscope the current signal or an average potential, after the average has been calculated. The arguments for the routine are:

F3(D/A starting address, counter low, counter high)

The contents of counter low through counter high are displayed on the oscilloscope. The D/A is 8 bits wide, which allows 256 values to be displayed at one time. If there are more than 256 counters to be displayed, the routine displays 256 evenly spaced counters.

Although the routine as presently used is for display on an oscilloscope, the routine can be modified to perform other functions. It could, for example, present a sine wave, or it could adjust stimulus conditions.

\section{F3(Mtape)}

The amount of data in analog studies requires an efficient recording mechanism. A routine was generated to dump the contents of certain counters onto magnetic tape. The routine involves:

F3(Mtape starting address, counter low, counter high)

The routine dumps the contents of counter low through counter high onto magnetic tape for later analysis.

\section{ANALOG DECISIONS}

Efficient routines have been created to make decisions based on the contents of counters. They are F3(If) and F3(Window). 


\section{F3(If)}

This routine compares the contents of one counter against the contents of another counter. A $\mathrm{Z}$ pulse is issued, depending on the outcome. The format is:

F3[If starting address, CX, CY, $\mathrm{Z}(\mathrm{CX}<\mathrm{CY})$, $\mathrm{Z}(\mathrm{CX}=\mathrm{CY}), \mathrm{Z}(\mathrm{CX}>\mathrm{CY})]$

An example of this may be seen in State Set 3 of Figure 3. If the contents of Counter 1 is less than that of Counter 2, Z7 is issued. If the contents of Counter 1 is equal to or greater than Counter $2, \mathrm{Z} 8$ is issued.

\section{F3(Window)}

Often decisions are based on several $A / D$ values. If the $A / D$ values are within a certain range, one transition occurs. If the $A / D$ values are not within that range, a different transition occurs. Although these decisions could be made by the F3(If) function, this would be inefficient. Thus, a window discriminator was generated, with the following format:

F3[Window starting address, number of windows, counter location for the first parameter of the first window, counter location for the first parameter of the second window, . ., Z(hit), $Z$ (miss)].

A $\mathrm{Z}$ (hit) is issed if the signal goes through all the windows. If the signal fails to pass through a window, a $\mathrm{Z}$ (miss) is issued.

Four counters are needed to give the parameters for the window. The first counter contains the counter value where the discrimination begins. The second counter contains the counter value where the discrimination ends. The third counter contains the minimum value of the window. The last counter contains the maximum value of the window.

An example of the F3(Window) routine is shown in State Set 3 of Figure 2. There is one window. The parameters are located in Counters 151 through 154. The values within the counters specified by Counters 151 and 152 are examined. If all the values are within the range indicated by Counters 153 and 154, a Z4 is issued; if not, then a Z5 is issued.

\section{ANALOG ANALYSIS}

Limited analysis can be performed on line. To date, means and standard deviations have been calculated on line. Other analyses are performed off line.

\section{F3(Math Pak)}

A general purpose, double precision, fixed point math package has been created. The package performs addition, subtraction, multiplication, division, and square root operations. There is also a DO loop. The usefulness of this package is limited by its inability to nest DO loops.

\section{F3(Sum)}

This routine has been generated to calculate double precision sum of scores and sum of squares of single precision values. The routine is used in conjunction with F3(means) to generate single precision means and standard deviations. The format of the routine is:

F3(Sums starting address, counter low, counter high, beginning counter of sum of scores, end counter of sum of scores, first counter of sum of squares)

The values to be calculated are in counter low through counter high. Since the sum of scores and sum of squares are double precision, twice as many counters as the values calculated are needed.

An example of this routine is shown in State Set 2 of Figure 1. The contents of Counters 1 through 150 are individually summed and squared. The sum of scores of Counter 1 is contained in Counters 151 and 152. The sum of scores of Counter 150 is in Counters 449 and 450. The sum of squares are in Counters 451 through 750.

\section{F3(Means)}

This routine takes the sum of scores and the sum of squares generated by F3(Sums) and calculates the means and standard deviations. The format is:

F3(Means starting address, first location of the sum of scores, last location of the sum of scores, number of trials, first address of the sum of squares)

The routine is shown in State Set 3 of Figure 1. The means are calculated for 100 trials, using the sum of scores located in Counters 151 through 450 . The means are calculated to the first decimal place. They are deposited in Counters 151 through 300 . The standard deviations are also calculated to the first decimal place. They are deposited in Counters 451 through 600 .

\section{PROGRAM UTILIZATION}

The routines described above were written to run a laboratory concerned with operant conditioning of neural events. The CAT program and the operant conditioning programs described below demonstrate typical usage of the F3s. The classical conditioning program illustrates a procedure described by Millenson et al. (1973) which previously could not be handled by SKED.

\section{The Return of the CAT}

Figure 1 gives the state notation for presenting a stimulus (S1) every $5 \mathrm{sec}$. The evoked potential is recorded on magnetic tape [F3(Mtape)]. The potential is recorded for $300 \mathrm{msec}$.

After 100 trials, the average evoked potential is calculated. This is the same function that the Computer 

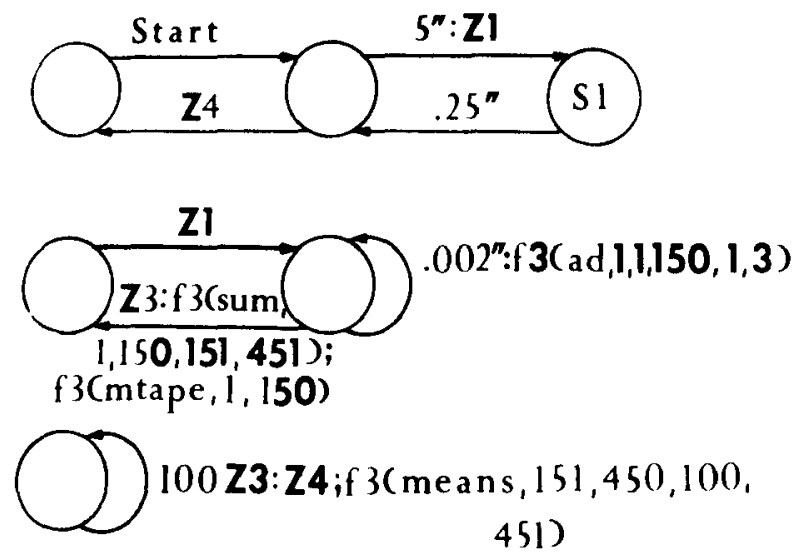

Figure 1. State notation to calculate the means and standard deviations of an evoked potential.

Average of Transients (CAT) performs. Unlike the CAT, the program depicted in Figure 1 also calculates the standard deviations.

\section{Operant Conditioning of Neural Events}

Figure 2 depicts the state notation for reinforcing an organism for emitting a specified neural signal. A stimulus ( $\mathrm{S} 1$ ) is presented every $5 \mathrm{sec}$. The signal is recorded for $300 \mathrm{msec}$, and it is put onto magnetic tape. If the signal is within the window, reinforcement (S2) is delivered.

\section{Classical Conditioning}

Figure 3 depicts a program that presents a CS and a UCS. The program records the latency of CR onset in Counter 5. The time that the CR peaks is recorded in Counter 4. The peak value of the $\mathrm{CR}$ is stored in Counter 1. The rest of the procedure as described by Millenson et al. (1973) can also be incorporated into state notation.

\section{CHANGES IN SKED}

\section{Open Command}

As is obvious by now, decisions are based on the contents of counters. To facilitate varying counters, an Open command has been added to the commands of SKED. The command allows the contents of counters to be varied under Teletype control. An example of the command format is as follows:

$$
\begin{aligned}
& 012 \\
& \emptyset \\
& \emptyset 128 \leftarrow 1 \\
& \emptyset 256 \leftarrow 512
\end{aligned}
$$

0 is the open command; 1 and 2 are the counters to be opened; $\emptyset$ is the box where the counters are located; 128 is the contents of Counter 1; and 256 is the
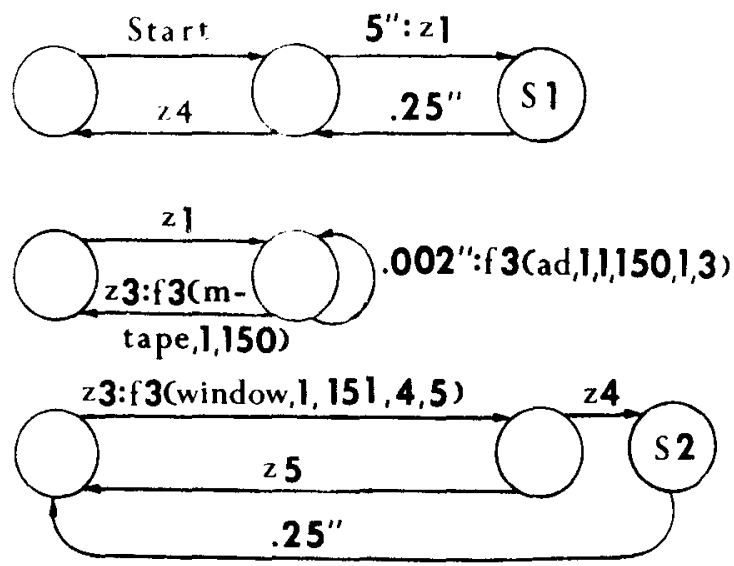

Figure 2. State notation to reinforce an organism contingent on a neural event.

contents of Counter 2.

A rubout $(/)$ leaves the contents of the counter the same. In the above example, the contents of Counter 1 remain the same, but the contents of Counter 2 are changed.

\section{Resolution}

SKED normally has a $10-\mathrm{msec}$ resolution for 10
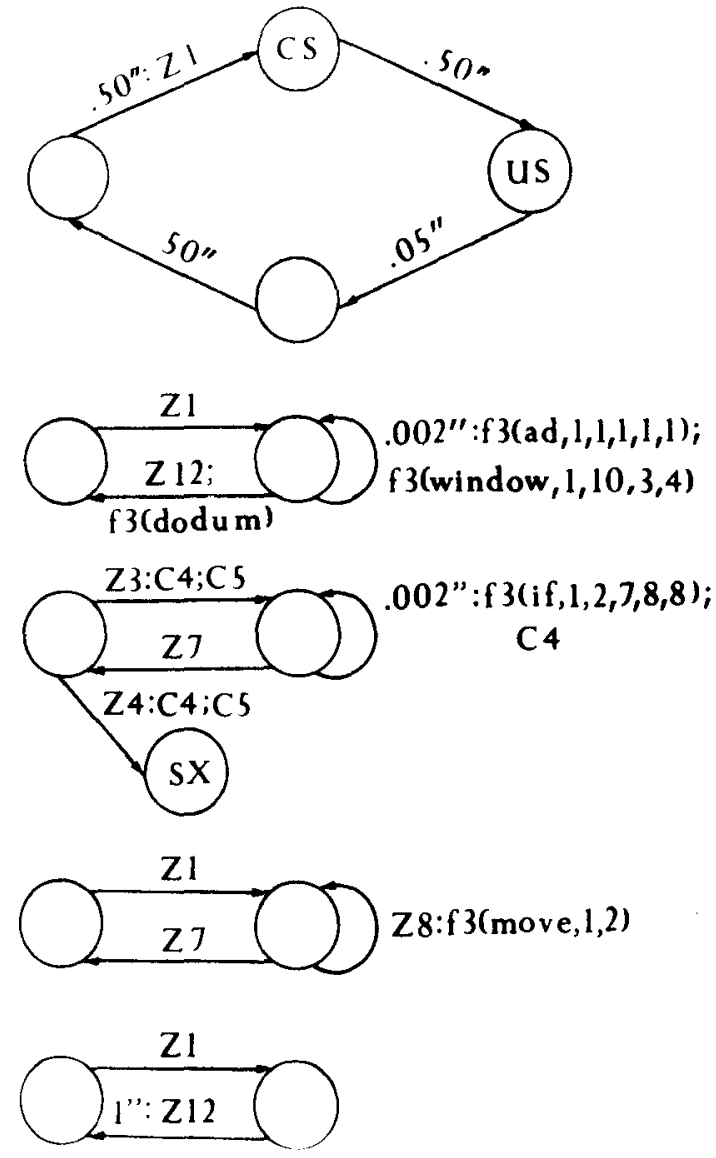

Figure 3. State notation depicting a classical conditioning procedure described by Millenson et al. (1973). 
boxes. A $1-\mathrm{msec}$ resolution can be used with a simple program and with a limited number of boxes. Thus, there is a trade off between the number of boxes and the resolution. In one laboratory, SKED has been modified to run just one box at a $1-\mathrm{msec}$ resolution. This trade off is acceptable in this situation, where memory is limited $(4 \mathrm{~K})$ and resolution is critical.

$$
15^{\prime} \rightarrow-\rightarrow \text { STOP }
$$

Use of the F3s described above reduces programming time and is efficient in the use of core. A program that would take 1 to 3 weeks to be programmed in assembly language requires $4 \mathrm{~h}$ in SKED. The CAT program runs in less than $700_{8}$ (excluding counters).

The routines are not limited to analog data. The F3(If), for example, can be used with discrete data.

The routines have been written for a special version of SKED. They have subsequently been rewritten for RTS4 and RTS8. They are available, without starting addresses, from the SKED Users Group .

\section{REFERENCE NOTES}

1. Butler, F. E. Use of SKED for classical and operant conditioning of single units of the rat's brain. Paper presented at the meeting of the American Psychological Association, Montreal, August 1973.

2. Kadden, R. M. Üse of SKED computer system for on-line data reconding of candiac conditioning experiments. Pape presented at the meeting of the American Psychological Association, Montreal, August 1973.
3. Ly on, D. O. An introduction to state diagramming as an aid for teaching complex schedules of reinforcement. Paper presented at the meeting of the American Psychological Association, Montreal, August 1973.

4. Michael, J. The use of the state system notation in teaching about behavioral experiments. Paper presented at the meeting of the American Psychological Association, Montreal, August 1973.

5. Snapper, A. G., Knapp, J. Z., Kushner, H. K., \& Kadden, R. M. A notation system and computer program for behavioral experiments. Paper presented at the meeting of the Digital Equipment Computer Users Society, New York, June 1967.

\section{REFERENCES}

Fowler, S. C. A minicomputer system for recording the dy namic properties of individual operant response. Behavior Research Methods \& Instrumentation, 1974, 6, 288-292.

Millenson, J. R. A programming language for on-line control of psychological experiments. Behavioral Science, 1971, 16, 248-256.

Millenson, J. R., Kehoe, E. J., Tait, R. W., \& Gormezano, I. A minicomputer program for control and data acquisition in classical conditioning. Behavior Research Methods \& Instrumentation, 1973, 5, 212-217.

Polson, P. G. SCAT: Design criteria and software. Behavior Research Methods \& Instrumentation, 1973, 5, 241-244.

Snapper, A. G. Use of a notational system for digital control and recording. Behavior Research Methods \& Instrumentation, $1973,5,124-129$.

Snapper, A. G., \& Hamilton, B. Programming special functions in the SKED system. Behavior Research Methods \& Instrumentation, 1974, 6, 173-176.

Snapper, A. G., Knapp, J. Z., \& Kushner, H. K. Mathematical description of schedules of reinforcement. In $W . N$. Schoenfeld (Ed.), The theory of reinforcement schedules. New York: Appleton-Century-Crotts, 1970. Pp. 247-275.

Snapper, A. G., \& Walker, A. The SKED software system. DECUS Program Library, DECUS 8-465, 1971.

Tait, R. W., \& Gormezano, I. A minicomputer program for stimulus control and analog data for discrete trial paradigms in biological preparations: Classical conditioning. Behavior Research Methods \& Instrumentation, 1974, 6, 296-300. 